http://jmscr.igmpublication.org/home/ ISSN (e)-2347-176x ISSN (p) 2455-0450 crossref DOI: https://dx.doi.org/10.18535/jmscr/v8i5.24

\title{
Prescribing Pattern of Anti Platelet Medications of Cardiovascular Disease Patients in Tertiary Care Teaching Hospital
}

\author{
Authors \\ Emil G Rajesh*1, Muhammed Jasim", Riya Ann Roy ${ }^{1}$, Sandra Alappattu', \\ Dr V Karthikeyan", Dr Neelakantan $\mathbf{V}^{2}$
}

${ }^{1}$ Department of Pharmacy Practice, Grace College of Pharmacy, Kodunthirapully, Palakkad, Kerala, India ${ }^{2}$ Professor and Head of Department of Medicine, Karuna Medical College, Vilayodi, Palakkad, Kerala, India *Corresponding Author Emil G Rajesh

\begin{abstract}
Background: Cardiovascular disease (CVD) is the name for the group of disorders of heart and blood vessels, and includes: hypertension (high blood pressure), coronary heart disease (heart attack), cerebrovascular disease (stroke), peripheral vascular disease, heart failure, rheumatic heart disease, congenital heart disease, cardio myopathies.
\end{abstract}

Aim: The aim of the study is to assess the prescribing pattern of anti-platelet medications for CVD patients.

Methodology: This is a prospective observational study conducted in the General medicine ward of Karuna Medical College Hospital from October 2019-april 2020. The useful data were obtained from patient case profiles, 236 cases were collected from general medicine wards, according to study criteria.

Results: In this study of 236 case sheets of patients with CVD were analyzed, out of that 160 (68\%) were males and 76 (32\%) were female. The distribution of CVD patients according to age groups 30-40,41-50, $51-60,61-70,71-70$ and $>80$ years are found to be 2\%, 11\%,28\%, 26\%,24\%, and 9\% respectively. The drug utilization of anti-platelets was found to be $84 \%$. Majority of the patients were prescribed with aspirin.

Conclusion: We conclude that minimizing the number of drugs per prescription, the irrational prescribing pattern of the drugs can be avoided by strictly following to the treatment guidelines and Ideal prescription writing.

Keywords: Anti-platelets, Cardiovascular disease, prescribing Pattern.

\section{Introduction}

CVD risk was found to be highest in the north eastern and southern states of the country, ranging from $13.2 \%$ in Jharkhand to $19.5 \%$ in Kerala. $^{2}$ As the active lifestyle and agrarian diet of India has changed to sedentary lifestyle and fast foods in shorter span of time, the fatality rate due to noncommunicable diseases like stroke, cardiovascular diseases and diabetes has been increased rapidly. ${ }^{3}$ CVDs are the number one cause of death globally: 
more people die annually from CVDs than from any other cause. An estimated 17.8 million people die from CVDs in 2017 of which more than three quarters were in low income and middle income countries. $^{4}$

Drug utilization is defined by World Health Organization (WHO) in 1977 as the "marketing, distribution, prescription and the use of drugs in a society, with special importance on the resulting medical, social and economic consequences. Drug utilization is essential for detecting the irrational use of drugs, making interventions to improve drug use and improve the quality of life. Drug utilization studies are powerful investigative tools to ascertain the role of drugs in determining the therapeutic efficacy, cost effectiveness and minimizing adverse effects. ${ }^{5}$

Antiplatelet agents are drugs that reduce the ability of platelet to engage in thrombus formation. They do so by reducing the ability of platelets to aggregate together and also by inhibiting other aspects of platelet function that collectively contribute to thrombus formation. ${ }^{6}$

Antiplatelet agents are well recognized for their beneficial impact on decreasing the frequency of ischemic events related to thrombus formation at the site of atherosclerotic plaque, fatal or nonfatal myocardial infarction, fatal or nonfatal ischemic stroke and other vascular - related death. However, it is important to distinguish these arterial thrombi from those that occur in the venous system without an underlying plaque and that are not particularly responsive to antiplatelet agents ${ }^{7}$. The study of prescribing pattern is a component of medical audit that does monitoring and evaluation of the prescribers as well as recommends necessary modifications to achieve rational and cost-effective medical care. Studies of prescribing patterns and drug utilization are useful to identify the problems and provide feedback to prescribers so as to create awareness about rational use of drugs. ${ }^{8}$ Clinicians face a demanding task when prescribing medications. Rational prescribing requires the practitioner to make an accurate diagnosis, understand the
Pathophysiology of the disease or condition, know the pharmacology of the drug prescribed, and consider the many others. Elements of appropriate medication use. ${ }^{9}$ The effectiveness studies reinforce the benefits of cardiovascular medications in routine clinical practice and highlight the importance of taking this medication as prescribed to optimize patient outcomes. ${ }^{10}$

\section{Methodology}

A prospective observational study was conducted in the department of general medicine at Karuna Medical College Hospital, Vilayodi, Chittur, Palakkad. This study was conducted for a period of six months (October 2019- April 2020). This present study was approved by the Institutional Human Ethics Committee of KMCH, Palakkad. Their data was collected from regularly from the General Ward of Medicine, without interfering with their treatment. Each patient age, sex, diagnosis (only cardiac patients) and prescribed generic and brand names of the drugs were recorded in the prepared case record form. The collected data was analyzed to study- route of administration of drugs, anti platelets prescribed for cardiac patients. Patients age greater than 30 years (Both male and female), those with established diagnosis of CVD, admitted in the ward of general medicine department and patients with co morbidities like diabetes mellitus, COPD, thyroid disorders, asthma CKD, anemia, GI disorders were included our study and pregnant women, patient who are not willing to participate in the study were excluded from our study. In prescribing analysis we used WHO core drug indicators in that, the average number of antiplatelet drug per encounter $=$ total no. of drugs prescribed/total number of encounters sampled, percentage of antiplatelet drugs prescribed by generic name $=($ Number of drugs prescribed by generic name/total number of drugs prescribed) $\mathrm{x}$ 100 , percentage of encounters with an antiplatelet drug prescribed $=($ Number of antiplatelet drugs prescribed/Total number of drugs prescribed) $\mathrm{x}$ 100 , percentage of encounters with an injection 
prescribed. $=($ Number of patients encountered during which an antibiotic or an injection are prescribed/total number encounters sampled) $\mathrm{x}$ 100 , percentage of drug prescribed from essential drug list of Formulary $=$ (Number of products prescribed which are listed on the EDL or local formulary [or which are equivalent to drugs on the list]/total number of products prescribed) x 100 were calculated by using these formulas. The collected data was analyzed in MS Excel and descriptive statistics were used for analyzing the result of the study.

\section{Result \& Discussion}

According to the inclusion criteria, we enrolled 236 participants in our study. Among the study population, $68 \%$ of male patients followed by $32 \%$ of patients from female, male patients were more prone to have cardiovascular disease when compared with female patients. Similar study was conducted by Kumar V S et al., (2019) also shows that the males are more prone to have cardiovascular disease than females ${ }^{11}$

Figure 1: Distribution of patients based on gender

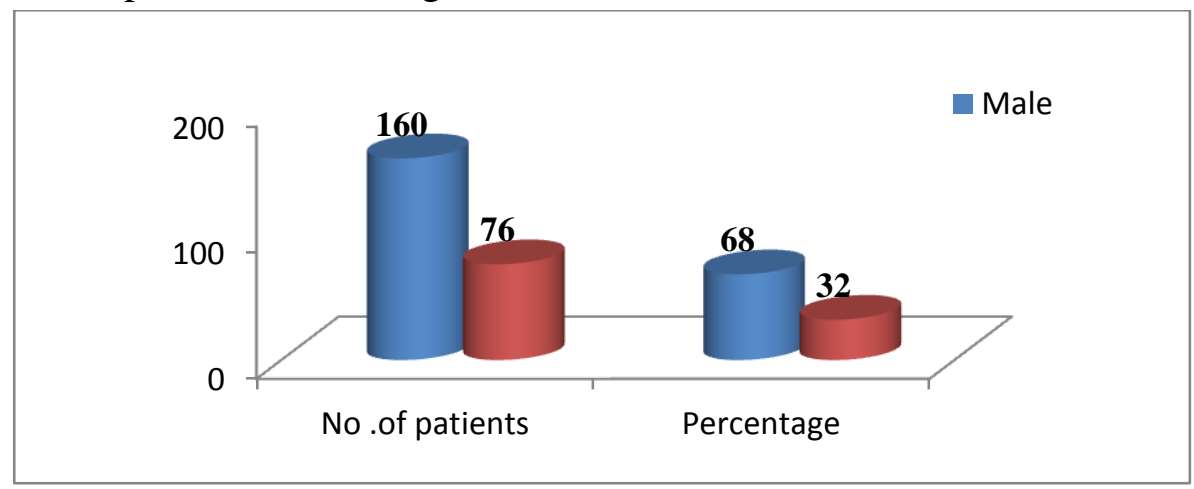

Age is an independent risk factor for developing CVD, the lifetime risk of CVD for an individual would continue to increase with age. However, the lifetime risk for CVD is lower at age 70 than at age 50 years, for an individual whose lifestyle risk factors remains unchanged. In our study population 129 patients were affected in the age group of 50 to 60 years.

Figure 2: Distribution of patients based on age groups

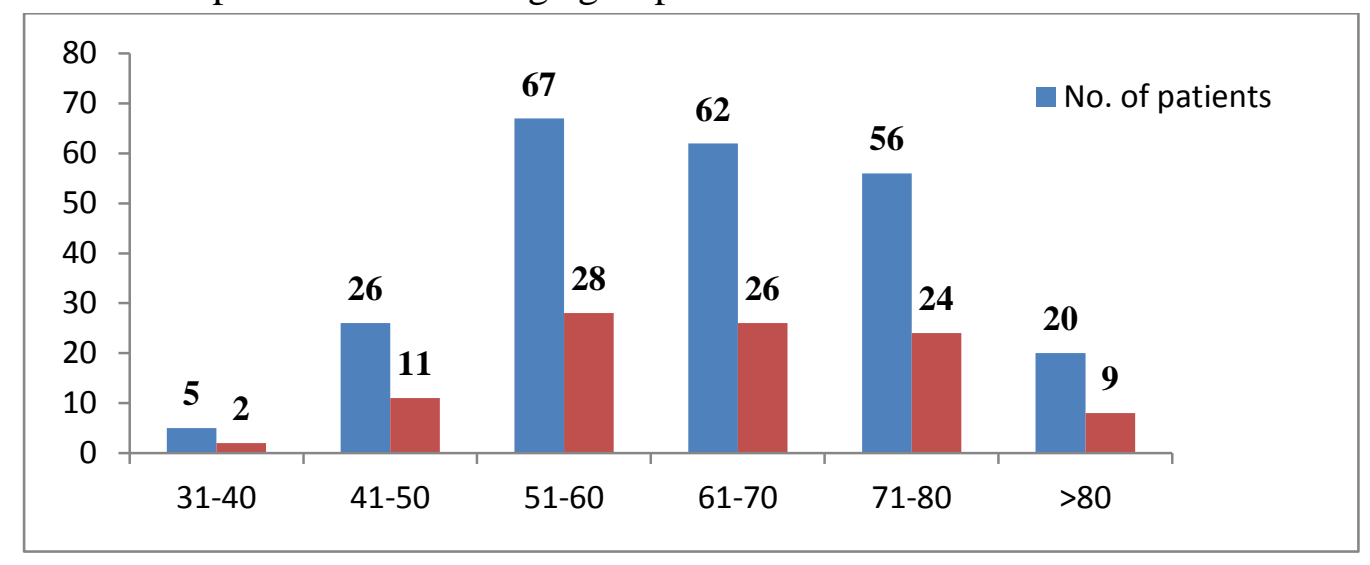

Tobacco and alcohol use interact to influence risk for cardiovascular disease is limited. Alcohol consumption of three or more drinks per day and cigarette smoking share similar, and probably additive, effects on some forms of cardiovascular disease. There is relatively little evidence, however, that the effects are worse when smoking and drinking occur together than would be expected from their independent effects. In most cases, moderate drinking does not share these 
risks and even has opposite effects of cigarette smoking on some risk factors. Based on the distribution of social history in 236 cases, majority of patients (136 patients) were not having any social history. While $15 \%$ of samples were smokers, $4 \%$ were alcoholics and $23 \%$ were both alcoholics and smokers. Similar study conducted in 2018 by Fathima et al., shows that bath alcoholics and smokers have increased number compeered with smokers and alcoholics ${ }^{12}$ Among the study population $42 \%$ of patients having more risk to cause CVD.

Figure 3: Distribution of patients based on social history

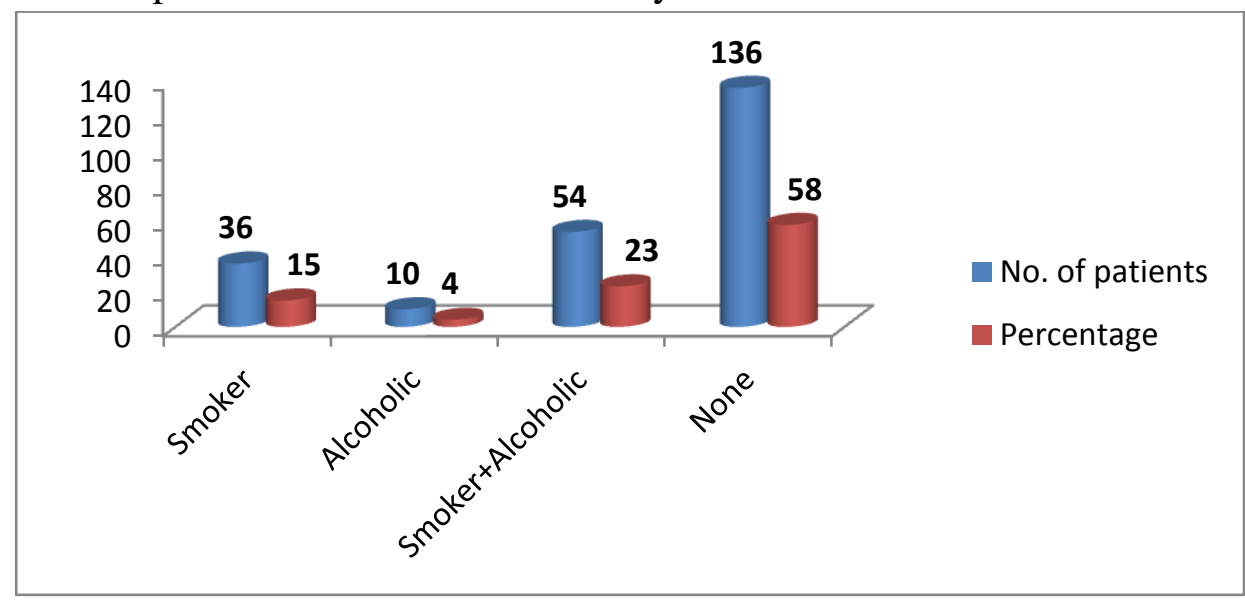

Co morbidity is a major public health issue, which challenges health care configured around single diseases. Aim To provide an overview of frequent disease combinations of one and two additional chronic diseases and groups among patients with cardiovascular disease (CVD) in general practice. In 236 cases collected from the general medicine ward, most of patient profiles is having co morbid conditions ,27\% of patients having diabetes, $5 \%$ having renal disorders, $2 \%$ of each having bronchial asthma and anemia, 6\% having COPD , $1 \%$ each having thyroid disorders, GI disorders and $0.4 \%$ of combination of diabetes and bronchial asthma, $15 \%$ having other conditions and the co morbid conditions of $40 \%$ patients are either not known or missing in the case sheets. In a similar study conducted by Fathima et al., in 2019 shows majority of patients having kidney disease followed by diabetes, asthma and thyroid disorders. $^{12}$

Figure 4: Distribution Based On Co-morbid Conditions.

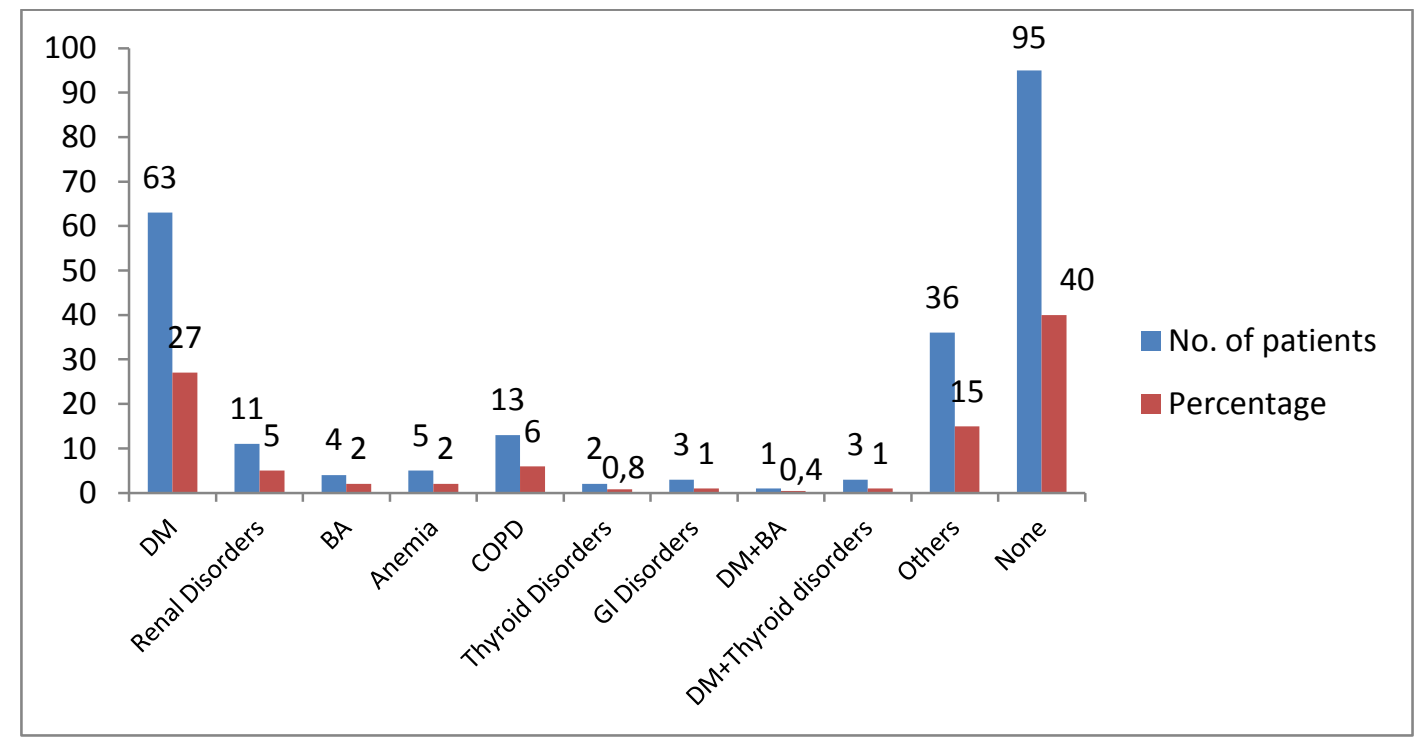


In our study 236 cases analyzed, which contains total number of 1720 drugs out of which 233 drugs were anti platelet drugs. Average number of drugs per patient is 7.28 and average number of anti platelet drugs per patient is 0.98. 33 prescriptions were written in generic name and
197 prescriptions were written in brand names. Similar study conducted in 2019 by Kumar VS et al., which contains 200 case sheets with an average number of anti platelet drugs per patient is 7.5 and $60 \%$ of drugs were prescribed in generic names. ${ }^{11}$

Table 1: The Prescription Analysis.

\begin{tabular}{|l|l|c|}
\hline S.NO. & PRESCRIPTION CATALOGUE & RESULTS \\
\hline 1 & Total no. of case sheets analyzed & 236 \\
\hline 2 & Total no. of drugs prescribed & 1720 \\
\hline 3 & Average no. of drugs per patient & 7.28 \\
\hline 4 & Total no. of anti platelet drugs & $\mathbf{2 3 3}$ \\
\hline 5 & Average no. of anti platelet drugs per patient & $\mathbf{0 . 9 8}$ \\
\hline 6 & Total no. of single anti platelet drug prescribed & 145 \\
\hline 7 & Total no. of anti platelet drugs prescribed orally & $\mathbf{2 3 3}$ \\
\hline 8 & Total no. of anti platelet drugs prescribed injectables & $\mathbf{0}$ \\
\hline 9 & Anti platelet drugs prescribed in generic name & 33 \\
\hline 10 & Anti platelet drugs prescribed in brand name & 197 \\
\hline
\end{tabular}

From the present observations, it was found that average number of drugs per prescription encounter was 7.28, which was justifiable even though it is more than WHO standard $(<2)$, as most of them are prescribed for cardiovascular emergencies. This cannot be considered as poly pharmacy as there is need of empirical therapy for management of life threatening conditions. The
WHO highly recommends physicians to prescribe the drugs in generic names. In this study, drugs prescribed by generic name were about $14 \%$. According to WHO standard for it was $100 \%$. Prescribing drugs from the EDL prepared by WHO means rational prescribing. All drugs in this study were prescribed from hospital formulary.

Table 2: Assessment of WHO Prescribing Indicators

\begin{tabular}{|l|c|}
\hline PARAMETERS ASSESSED & RESULTS \\
\hline Average no. of drugs per prescription & 7.28 \\
\hline Percentage of drugs prescribed by generic name & 14 \\
\hline Percentage of encounters with an antiplatelet drug prescribed & 84 \\
\hline Percentage of encounters with an antiplatelet injection & 0 \\
\hline Is the drugs prescribed are from the hospital formulary & YES \\
\hline
\end{tabular}

According to the utilization study of antiplatelet drugs in 236 samples, majority of patients were prescribed with aspirin in both males (62\%) and females $(41 \%)$ followed by other categorises. In females ,aspirin +atorvastatin+ clopidogrel combination is of $34 \%$, aspirin +atorvastatin is of $20 \%$, followed by $1 \%$ in clopidogrel, aspirin +clopidogrel, aspirin along with aspirin + atorvastatin. In males $12 \%$ was found in aspirin + atorvastatin, $14 \%$ in aspirin+atorvastatin +clopidogrel combination. In a similar study conducted by Muneeshwar et al., in 2018 aspirin is having the highest rate of prescription compared with other antiplatelet drugs. ${ }^{13}$

Table 3: Shows Sample Distribution According to Anti-platelet Therapy.

\begin{tabular}{|l|c|c|}
\hline $\begin{array}{l}\text { Anti platelet drug and/or } \\
\text { combinations. }\end{array}$ & $\begin{array}{c}\text { Male } \\
(\%)\end{array}$ & $\begin{array}{c}\text { Female } \\
(\%)\end{array}$ \\
\hline Aspirin & $\mathbf{6 2}$ & 41 \\
\hline Clopidogrel & 4 & 1 \\
\hline Aspirin + Clopidogrel & 1 & 1 \\
\hline Aspirin, Clopidogrel & 4 & 1 \\
\hline Aspirin + Atorvastatin & 12 & 20 \\
\hline $\begin{array}{l}\text { Aspirin, } \\
\text { Aspirin + Atorvastatin }\end{array}$ & 1 & 1 \\
\hline Aspirin+ Atorvastatin+Clopidogrel & 14 & 34 \\
\hline Aspirin+ Rosuvastatin +Clopidogrel & 2 & 1 \\
\hline
\end{tabular}




\section{Conclusion}

The present study revealed that, it was observed the incidence of CAD was more common in male compared to female and the risk for coronary artery disease increased with increase in the age. The

maximum number of patients was male; it may be due to smoking and alcoholic habits. In this cardiovascular disease, 51-60 age group patients have shown more prevalent when compared to that of other age groups. The most frequently prescribed drugs group is anti platelet. By prescribing the generics patients can be able to economize the expenses spent on the healthrelated problems. Before prescribing to the patients, evaluation of medications with suitable criteria is required. By minimizing the number of drugs per prescription, the irrational prescribing pattern of the drugs can be avoided by strictly following to the treatment guidelines and Ideal prescription writing. So, the treatment will be effective, for the recovery of the normal life of the patient.

\section{Reference}

1. WHO-About cardiovascular diseases [Internet] 2020[cited on 2019 sept 15]. Available from: http://origin.who.int/cardiovascular_disease s/about_cvd/en/

2. Kerala at highest risk for cardiovascular diseases in India, finds a study. The news minute [Internet] 2016 jun 21 [cited on 2019 sept].Available from: https://www.thenewsminute.com/article/ker ala-highest-risk-cardiovascular-diseasesindia-finds-study-83460.

3. M Reddy, T Prasad.A Study on antiplatelets and anticoagulants utilisation in a tertiary care hospital.2018:10(5);155-161.

4. World Health Organization. Global Status Report of NCD 2010.Geneva: World Health
Organization.

Available

from:

https://www.who.int/nmh/publications/ncd_ report_full_en.pdf.Accessed 4.6.13

5. Abrams WB (Ed.). Workshop on drug utilization review. Clinical Pharmacology and Therapeutics 50(5 Part 2 Suppl.): 593640, 1991

6. Wolf Z, Mani H, Lindhoff-Last E. Significance of platelet function tests. Internist 2010; 51: 1095-6

7. Albert ferro, David A. Garcia, et al. Antiplatelet and Anticoagulation Therapy., 2013; 122-440.

8. Shruthi D, Venkateshwarlu K, et al. Prescribing Pattern in Coronary Artery Disease: A Prospective Study. IJPRR., 2014; 3(3): 24-33.

9. J Hanlon, K Schamader, G samsa. A method for assessing drug therapy appropriateness .J Cila Epidemiol.1992,45(10):1045-1051.

10. P Michael, C Bryson. Medication Adherence: Its Importance in Cardiovascular Outcomes. Circulation 2009: 119(23);3028-3035.

11. Kumar V S, Jyothi P D et al., Assessment and Drug Utilization Pattern on Antiplatelet Agents in Cardiovascular Patients - A Prospective Study in Tertiary Care Hospital. International Journal Of Pharmacy And Pharmaceutical Research. 2019: 14 (2);109119.

12. Fatima S, Beegum S.P rescription pattern of anti-hyperlipidemic and anti-platelt drugs in the management of CAD in a tertiary care hospital. World journal of pharmacy and life sciences.2019:5(3); 112-121.

13. Muthuvel T, Vipin V. A Prospective Study On Drug Utilization And Evaluation Of Prescribing Pattern Of Drugs Use In Patients Of Myocardial Infarction. .World Journal of Pharmacy And Pharmaceutical Research.2018:8(3); 615-633. 